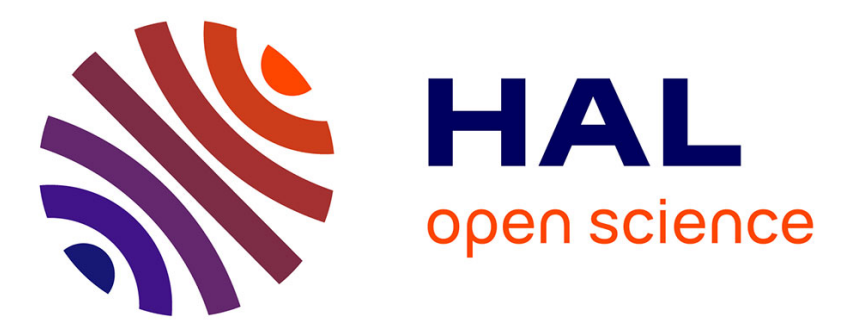

\title{
Troubadours et analyses factorielles : approches statistiques à la représentation de l'auteur dans les chansonniers occitans $\mathrm{A}$, I et $\mathrm{K}$. \\ Jean-Baptiste Camps
}

\section{- To cite this version:}

Jean-Baptiste Camps. Troubadours et analyses factorielles : approches statistiques à la représentation de l'auteur dans les chansonniers occitans A, I et K.. Nouvelle Recherche en domaine occitan: approches interdisciplinaires, Jun 2009, Albi, France. halshs-00824016

\section{HAL Id: halshs-00824016 \\ https://shs.hal.science/halshs-00824016}

Submitted on 20 May 2013

HAL is a multi-disciplinary open access archive for the deposit and dissemination of scientific research documents, whether they are published or not. The documents may come from teaching and research institutions in France or abroad, or from public or private research centers.
L'archive ouverte pluridisciplinaire HAL, est destinée au dépôt et à la diffusion de documents scientifiques de niveau recherche, publiés ou non, émanant des établissements d'enseignement et de recherche français ou étrangers, des laboratoires publics ou privés. 


\section{TROUBADOURS ET ANALYSES FACTORIELLES : APPROCHES STATISTIQUES À LA REPRÉSENTATION DE L'AUTEUR DANS LES CHANSONNIERS OCCITANS $A, I$ et $K$.}

\section{Introduction}

Parmi les chansonniers datant des XIII ${ }^{\mathrm{e}}$ et $\mathrm{XIV}^{\mathrm{e}}$ siècles qui nous sont parvenus, neuf sont dotés de miniatures, à des degrés variables ${ }^{1}$. Joseph Anglade, probablement un des premiers à s'y intéresser, en recensait déjà 7 , dans un article daté de $1924^{2}$. Cet article se voulait une étude préparatoire à une monographie, l'Iconographie des Troubadours, qui n'a jamais, semble-t-il, vu le jour. Depuis, le sujet des miniatures des chansonniers occitans est longtemps resté en friche. Du moins jusqu'au renouveau de l'étude des chansonniers dans les années 1980, au cours desquelles de nombreux philologues tels que Maria-Luisa Meneghetti, Angelica Rieger et Geneviève Brunel-Lobrichon se sont penchés sur le sujet ${ }^{3}$. Plus récemment, l'étude de ce sujet a pris son envol, avec la parution assurée par Françoise Vielliard et Jean-Loup Lemaître de la série des Portraits de Troubadours, qui permet enfin un accès aisé à ce corpus, ainsi qu'avec les études d'historiens de l'art comme Giordana Mariani Canova ${ }^{4}$.

Les miniatures contenues dans ces chansonniers sont de deux types : initiales historiées ( $A I K$ et $N$ ) et miniatures indépendantes de l'initiale ( $M$ et $H$ ), auxquelles s'ajoutent les dessins marginaux, ajouts plus tardifs présents dans le chansonnier $N$ et qui forment ce que Rieger qualifie de "bande-dessinée $»^{5}$. Le corpus est loin d'être homogène, et les miniatures des différents chansonniers répondent à des projets différents. De nombreux critères permettent de les différencier, notamment du point de vue des rapports qu'entretient l'image avec le texte et avec le contexte général du manuscrit.

Tout d'abord, six de ces chansonniers sont vénètes (ou de modèle vénète pour $M$ ), tandis que trois sont originaires du Languedoc. Les chansonniers languedociens, plus tardifs, possèdent un programme iconographique qui, quoique non dénué de liens avec le texte, " appare molto meno nitido $»^{6}$ que celui des chansonniers vénètes, et mêle des représentations humaines à des représentations végétales et animales, réalistes ou fantastiques. Qui plus est, ils n'associent pas les miniatures aux vidas qui sont soit contenues dans des sections à part, soit absentes. Les chansonniers vénètes, quant à eux, sont les seuls chez lesquels on rencontre l'association entre des miniatures qui se rapprochent le plus de ce que nous pourrions qualifier de portraits, et des vidas, les deux étant placés en tête de section en introduction aux textes de chaque troubadour.

$C^{\prime}$ est la disposition des chansonniers $A, I$ et $K$ qui pourraient provenir de Venise même ${ }^{7} . I$

1 Il s'agit des chansonniers $A, I, K, N, H$ (Vénétie, dernier quart du XIII ${ }^{\mathrm{e}}$ siècle) $M$ (Naples, fin XIII $\left.{ }^{\mathrm{e}}\right) ; E, C, R$ (Languedoc, XIV $\mathrm{V}^{\mathrm{e}}$ siècle), auxquels, pour être vraiment complet, il faudrait ajouter au moins un fragment (fragm. Romegialli). Le chansonnier $B$ n'a, lui, pas reçu de miniatures en dépit de l'espace réservé et les fragments $K^{a}$ et $\psi\left(K^{\prime}\right.$ et $K^{\prime \prime}$ pour F. Zufferey) ne portent aucune décoration (on observe cependant des espaces réservés, allant jusqu'à 6 lignes de réglure dans $\psi$ ), tandis que $A^{a}$ ( $A^{\prime}$ pour Zufferey) porte, au début de la section consacrée à Albertet et suite à sa vida, une grande initiale qui n'a toutefois reçu qu'un décor végétal et géométrique ; Zufferey (1987 p. 322-331).

2 Il s'agit des chansonniers $A, H, L, I, K, M$ et $C$, laissant de côté $R$ et $N$; Anglade (1924).

3 Meneghetti (1984), Rieger (1985) et Brunel-Lobrichon (1991).

4 Lemaître et Vielliard (2006 et 2008) et Mariani-Canova (2004 et 2008) ; l'idée d'une recherche sur la représentation du troubadour comme auteur est en bonne partie redevable à Laura Kendrick (2001).

5 Rieger (1985 p. 387).

6 Meneghetti (1992 p. 334).

7 L'hypothèse d'une origine vénitienne, proposée par François Zufferey (1987, p. 65) sur la base de la place faite à Bertolome Zorzi dans ces trois chansonniers, est soutenue notamment par Giordana Mariani-Canova (2004, 
et $K$ proviennent d'ailleurs assez vraisemblablement du même atelier et présentent entre eux de grandes similitudes, au point qu'on a pu les qualifier de chansonniers jumeaux. $A, I$ et $K$ ont également en commun de dater du dernier quart du XIII ${ }^{\mathrm{e}}$ siècle ${ }^{8}$ et d'être des chansonniers de grand format, et contenant une récolte de textes assez structurée, avec peut-être une certaine volonté d'exhaustivité et de conservation. Pour ce qui est des miniatures, $A$ en comporte 45, $I$, 92 et $K, 78$. Leur origine géographique et chronologique, leur mise en page, la disposition de leurs vidas, et le type de leurs miniatures font de $A, I$ et $K$ les exemples les plus caractéristiques d'un modèle particulier de chansonniers.

Ce modèle répond à un projet bien défini, à une certaine considération de la poésie occitane et des troubadours, à la particularité du contexte vénète. En effet, si nous pouvons aujourd'hui parler des cansos de Giraut de Bornelh ou des sirventés de Bertran de Born, c'est que nous avons hérité d'une répartition des œuvres de la lyrique occitane par genre et par auteur, qui est en grande partie tributaire de la structure de ces grandes anthologies que sont les chansonniers. Compiler un chansonnier, c'est également trier, organiser, opérer des choix, ainsi que se préoccuper de l'attribution des textes (on sait la faible proportion de textes anonymes dans la lyrique occitane). Cette préoccupation biographique et cette volonté de structurer et d'encadrer les textes sous-tendent une vision particulière de ce qu'est la poésie des troubadours, de ce que sont les troubadours eux-mêmes.

Au-delà de la répartition des œuvres entre trois sections, consacrées aux cansos, tensos et sirventes, et du classement par auteur au sein de chaque section, les chansonniers vénètes présentent un certain nombre de caractéristiques remarquables, caractéristiques déterminées en bonne part par la situation même de la Vénétie qui est, au vu de la zone d'origine de la poésie d'oc, « un area laterale e conservativa di frontiera ${ }^{9}$, pour reprendre l'expression de Folena. Le décalage à la fois chronologique et géographique crée un besoin d'explicitation, d'interprétation, d'encadrement des textes de troubadours. Il a fallu donner aux lecteurs les clés de textes qui ne sont plus d'accès aisé, s'ils l'ont jamais été. Ce besoin est sans doute l'un des facteurs qui expliquent la faveur dont ont bénéficié les vidas. En effet, ces courts textes biographiques cherchent, en retraçant la vie d'un troubadour, à expliciter son œuvre, à en donner une interprétation. Sans s'attarder sur la problématique de l'origine de ces vidas, on remarquera tout d'abord que leur contenu s'inspire très largement des œuvres des troubadours qu'elles concernent, cherchant dans les poésies des détails biographiques. Ces éléments donnent une consistance nouvelle à la notion d'auteur, servent à leur tour à fixer une lecture, une interprétation des œuvres du troubadour, dans ce que Laura Kendrick qualifie de « circularité herméneutique $»^{10}$.

En effet, les auteurs des vidas et les miniaturistes, étant à la fois récepteurs de textes déjà existants et inventeurs, mêlent ainsi interprétation et imagination, dialoguent avec les textes, les comprenant d'après leurs propres critères, ceux de leur milieu et de leur époque. Ils les transforment ainsi, leur donnent un autre sens, les 'recréent' pour ainsi dire, tout cela en voulant fixer, établir, un sens qui puisse les expliciter, fonder une interprétation du texte. L'importance de cette volonté biographique se manifeste matériellement par la vida, à l'encre rouge, et la miniature au début de chaque section d'auteur, ainsi que par la reprise du nom de l'auteur, encore à l'encre rouge, avant chaque œuvre. Ces éléments, encadrant le texte, cherchent à guider le regard du lecteur, à lui fournir un cadre qui guide sa compréhension des

p. 237 et 2008) et Fabio Zinelli (2007, p. 8).

8 Des éléments internes aux chansonniers permettent d'affiner cette datation. La présence des auvres de Bertolome Zorzi rend les trois chansonniers vraisemblablement postérieurs à 1273, en outre, la présence dans $I$ du cycle de sirventes concernant la guerre franco-aragonaise de 1285 (à la fin de la section des cansos, f. 149v150 ) fournit pour ce dernier un terminus post quem viable.

9 Folena (1990, p. 2).

10 Kendrick (2001, p. 513). 
poésies, et constituent ce que Maria-Luisa Meneghetti nomme «un doppio filtro metatestuale $»^{11}$.

Au-delà de la volonté d'expliquer le texte, il est possible que l'on ait cherché à doter les troubadours d'une dignité nouvelle, celle d'auteurs. En effet, en expliquant l'œuvre par la vie de celui qui l'a composée, en cherchant ce qui le caractérise, ce qui le différencie des autres, on s'éloigne de la pratique médiévale classique du texte cherchant à 'cacher' son auteur pour atteindre à l'universalité. On donne à des auteurs vernaculaires une dignité et un statut qui jusque là leur échappait et leur figure, matérialisée dans le manuscrit, peut servir de caution aux textes présentés, le troubadour faisant presque figure d'autorité, dans un contexte où la lyrique occitane devient l'affaire d'érudits, de spécialistes, de maîtres es trobar.

On est toutefois en droit de se demander ce que recouvre exactement cette notion de «portraits » qui, assurément, diffère de la nôtre. Dans la conception du portrait telle qu'en vigueur au XIII ${ }^{\mathrm{e}}$ siècle, on ne cherche pas à représenter une personne par des caractères physiques renvoyant à son apparence individuelle, mais on cherche plutôt à représenter son essence, sa qualité, par des attributs qui y renvoient. Ce fait est encore renforcé par la distance chronologique qui sépare le moment où les troubadours ont vécu de celui où on les a peints. Ce qui pourrait nous paraître une difficulté (l'absence de modèle vivant) n'en est pas vraiment une pour les miniaturistes. Mais alors que peint-on et quelle ressemblance cherche-t-on ?

En réalité, on cherche, par le biais de l'image, à transcrire un certain nombre de caractéristiques dont on affuble les troubadours. Pour ce faire, l'image dispose de son propre vocabulaire, qui se décline sur le mode de la variance autour de quelques grandes figures. C'est ce qui crée un sentiment de répétitivité, lorsque l'on aborde ces miniatures dans leur ensemble : elles ne sont jamais identiques, mais jamais vraiment différentes non plus.

Ce qui vient d'être dit de l'image peut aussi s'appliquer, dans une certaine mesure, au texte des biographies. On sait que les auteurs des vidas ne craignent pas d'inventer. Pour autant, cette inventivité n'est sans doute pas perçue comme fictionnelle ou mensongère. Le texte, autour d'un certain nombre de qualités, de formules et de motifs narratifs tisse une vie et un caractère qui correspondent à la nature de chaque troubadour telle qu'elle est alors perçue.

Je vais à présent tenter, tout d'abord, de prouver l'existence de correspondances entre l'image et le texte, entre leurs vocabulaires ; puis, je tracerai, à grands traits, la structure de ces deux vocabulaires tout en présentant la méthode employée pour les analyser ${ }^{12}$.

\section{Lien entre texte et image}

Un certain nombre de points peut nous amener à croire en l'existence d'un lien fort entre texte et image, notamment, dans un premier temps, au travers de l'analyse ponctuelle de certaines correspondances. Dans certains cas, en effet, la vida du troubadour, porteuse d'un épisode marquant ou évocateur, donne son sujet à la miniature. Ces cas, assez aisés à repérer, sont généralement assez fameux. À titre d'exemple, on pourra évoquer le cas de Jaufré Rudel qu'une miniature du chansonnier $I$ (f. $121 \mathrm{v})^{13}$ représente mourant dans les bras de la comtesse de Tripoli, comme le dit la vida, racontant cette histoire emblématique de l'amor de lonh, d'un chevalier traversant la mer par amour pour une dame qu'il n'a jamais vue :

E per voluntat delleis vezer, el se croset e se mes en mar, e pres lo malautia en la nau, e fo condug a Tripoli, en un alberc, per mort. E fo fait saber a la comtessa et ella venc ad el, al son leit e pres lo antre sos bratz. E saup qu'ella era la comtessa, e mantenant recobret

11 Meneghetti (1992, p. 348).

12 Cette méthode, que j'expose ici brièvement et dont je présente quelques résultats, est celle que j'emploie pour mon mémoire de Master II ; Camps (2009).

13 Lemaître et Vielliard (2006, p. 11 et n5). 
l'auzir el flairar, e lauzet Dieu que l'avia la vida sostenguda tro qu'el la agues vista ; et enaissi el mori entre sos bratz ${ }^{14}$.

Parfois, l'allusion se limite à une seule phrase, tirée de la vida, comme pour Giraut de Bornelh $(I, \mathrm{f} .14 \text { et } K, \mathrm{f} .4)^{15}$, représenté accompagné des ses « dos cantadors que cantavon las soas chansos $\gg^{16}$ ou à un mot, comme pour Peire de Valeria $(I, \mathrm{f} .122)^{17}$, qui tient dans sa main droite ce qui ressemble à une fleur. S'il porte cette fleur, c'est fort probablement car dans sa vida le mot de «flors » apparaît : «e fez vers (...) de foillas et de flors, e de cans e de ausels $»^{18}$.

Ces miniatures un peu particulières ne forment guère, toutefois, que la partie émergée de l'iceberg. Une fois celles-ci mises de côté, le reste présente un aspect assez uniforme, dans lequel on voit se dégager quelques grandes figures, sans pouvoir encore clairement établir une typologie précise ou des correspondances exactes. En effet, la plupart des miniatures semble se construire au travers de motifs récurrents qui se rapportent à des mots précis du texte. C'est ce que ces cas isolés ont le mérite de nous démontrer.

\section{Le vocabulaire du texte}

Portons maintenant notre attention sur la structure des vidas. Lorsqu'on les compare les unes aux autres, celles-ci présentent un grand nombre de similarités dans leur construction, dans leurs formules, dans leurs désignations des troubadours. On y trouve un certain nombre de termes et de formules récurrentes. Ce phénomène s'observe le mieux pour leur première phrase, qui se construit généralement selon un schéma : [Nom du troubadour] si fo de [lieu] $e$

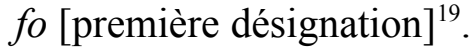

Cette première désignation peut souvent être qualifiée de désignation 'sociale'. En effet, on trouve, dans une large majorité des vidas, un terme renvoyant au rang du troubadour, soit par sa naissance (la formule $e$ fo fills de est très fréquente), soit directement. Elles peuvent être rassemblées en un certain nombre de catégories : serviteurs et personnages de basse extraction (sirven, sartres, forniers,...) ; bourgeois et marchands (borges, mercadiers,...); jongleurs (joglars); pauvres chevaliers (paubres cavalliers); chevaliers (cavalliers); châtelains (castellans); barons et grands nobles (gentils bars, vescoms, coms, princes, marques...); rois (reis); dames (domna, dompna); maîtres (maistres); chanoines (canorgues); clercs (clercs, clergues) ; moines (monges); évêques (evesques).

Cette désignation est généralement suivie par un certain nombre de formules, récurrentes, évoquant les qualités du troubadour, c'est-à-dire ses qualités personelles ou dans l'exercice de ses activité poétiques, et souvent une évocation des genres qu'il a pratiqués. Enfin, on trouve assez fréquemment également un motif narratif, qui cherche généralement à expliquer ce qui a mené le personnage à pratiquer l'art du trobar, et qui peut, par exemple, se rapporter à un épisode amoureux, source d'inspiration (c'est là le cas le plus fréquent) ou aux conditions matérielles qui ont poussé le personnage à se faire jongleur. Ces éléments semblent être en grande partie conditionnés par la première désignation du troubadour, désignation que j'ai qualifiée de « sociale ».

En effet, à certaines désignations semblent correspondre certaines formules. Par exemple, la formule, assez fréquente « bons cavalliers fo d'armas » est plus fréquente chez les castellan

\footnotetext{
14 Boutière (1964, p. 16-18); je reproduis ici le texte de $I$ avec ses variantes graphiques, même surprenantes. 15 Lemaitre et Vielliard (2006, p. 16-17 et $\left.n^{\circ} 8\right)$.

16 Boutière (1964, p. 39).

17 Lemaître et Vielliard (2006, p. 9 et $\left.\mathrm{n}^{\circ} 4\right)$.

18 Boutière (1964, p. 14); texte de $I$.

19 Ce «standard pattern » a été souligné par Elizabeth Wilson Poe (1984, part. p. 30-31).
} 
ou les baron $^{20}$ ), tandis que la formule « savis hom de letras et de sen natural » se retrouve chez les personnages qualifiés de maistres ou de canorgues $^{21}$.

Cette désignation semble également influer sur les motifs narratifs, ce que remarquait déjà Meneghetti, qui dressait une typologie de ces motifs centrée autour de la relation amoureuse et du succès, ou de l'insuccès, de celle-ci, qui serait fonction du rang social du troubadour ${ }^{22}$. Elle remarquait également que de ce rang social dépendait la place accordée à l'activité troubadouresque, qui passe au second plan lorsque le troubadour, l'amant, est de rang social équivalent à sa dame. Aux troubadours pauvres, donc, les histoires d'amour un peu tragiques, dans lesquelles la dame se refuse à leur accorder le plazer d'amor tandis que leur rôle se limite à étendre leur réputation, vanter leur beauté, et somme toute accroître leur pretz ${ }^{23}$.

Dans le chansonnier $A$, on retrouve ces désignations dans le texte des postilles, recommandations laissées à l'intention du miniaturiste, ce qui peut nous laisser penser que l'image se construit également autour d'un certain nombre de ces figures. Une autre preuve nous est apportée, paradoxalement, par les miniatures représentant Aimeric de Belenoi dans $I$ et $K(I, \mathrm{f} .125 \mathrm{v} \text { et } K \text {, f. } 111)^{24}$, dont la vida nous dit " clercs fo et fez se joglars $)^{25}$. En effet, le miniaturiste de $K$ a choisi de le représenter portant la tonsure, tandis que celui de $I$ a opté pour un personnage qui, même s'il ne porte pas d'instruments de musique, s'apparente plutôt par ses vêtements (notamment la coiffe et le chaperon) aux plus pauvres des laïcs non-nobles.

\section{Analyses factorielles et vocabulaire de l'image}

Une fois établie la correspondance entre le vocabulaire du texte et celui de l'image, il est nécessaire de décomposer l'image et ses éléments pour en analyser la structure. Pour cela, le relevé systématique d'un certain nombre de caractères de chaque image et leur mise en série les unes par rapport aux autres et par rapport au texte des vidas qui les accompagnent sont nécessaires. Pour cette mise en série l'outil informatique et la statistique sont riches de possibilités. Les analyses factorielles, notamment, constituent un instrument puissant pour l'étude de ce type de données ${ }^{26}$. J'ai retenu, pour la démonstration qui va suivre, 16 caractères qui sont la désignation dans la vida, les formules employées, l'âge, l'orientation des personnages, leur position, leurs gestes et la position de leurs mains, la barbe, la coiffe, le vêtement et la présence de fourrure, les éléments de décor, les accessoires, l'écu, la housse de cheval et les armoiries.

Je vais à présent tenter d'illustrer brièvement ma méthode par un exemple précis, celui des troubadours représentés en armes dans le chansonnier $I$, chansonnier dans lequel les nobles

20 On la retrouve notamment dans les vidas de Pons de Capduoill, du « vescoms de Saint Antoni » Raimon Jordan, de Guillems de Saint Ledier, tandis que Dalfin d'Alvernhe est qualifié comme étant le «meiller d'armas », voir Boutière (1964, respectivement p. 311, 159, 271 et 284).

21 On retrouve cette formule dans les vidas de Giraut de Bornelh, Daude de Pradas, Peire Rogiers et, sous une forme un peu différente, chez Peire d'Alverne, voir Boutière (1964, resp. p. 39, 233, 267 et 263).

22 Meneghetti (1992, p. 264).

23 Il est rare en effet qu'une dame de haut rang consente à s'accorder à quelqu'un qui lui soit inférieur, et si jamais cela se produit, elle en est en général blâmée, voir notamment la vida de Peire Rogier, Boutière (1964, p. 267). En revanche, elles ne répugnent pas à voir ces troubadours vanter leurs mérites, quitte à les 'duper', voir notamment la vida de Raimon de Miraval, Boutière (1965, p. 375-376, § 6-7).

24 Lemaitre et Vielliard (2006, p. 66-67 et $\left.\mathrm{n}^{\circ} 36\right)$.

25 Boutière (1964, p. 255).

26 Ces dernières permettent en effet de calculer les distances séparant chaque miniature, chaque modalité de chaque caractère - la façon de calculer ces distances variant d'un type d'analyse factorielle à une autre (analyse en composantes principales, analyse factorielle des correspondances, etc.) d'après des principes mathématiques qu'il serait délicat d'exposer entièrement ici — puis de projeter ces points et les distances qui les séparent dans un espace à $n$ dimensions. On recherche ensuite l'hyperplan séparateur permettant de répartir le mieux possible les miniatures. 
constituent environ la moitié du corpus et qui a la particularité de contenir de nombreuses représentations de troubadours en chevaliers, avec une grande diversification.

Si l'on examine, comme première vision d'ensemble, le graphique (fig. l) représentant la répartition des modalités sur un espace factoriel au terme d'une analyse des correspondances, plusieurs constatations semblent déjà s'imposer. Tout d'abord, l'existence de groupes biens distincts, un en haut à droite du graphique, et l'autre sur sa gauche. Si l'on examine de plus près ces deux groupes, on se rend compte que l'un d'entre eux contient les troubadours désignés comme nobles dans les vidas (paubres cavalliers, cavalliers, castellans, bars, etc) ainsi qu'un certain nombre de leurs attributs, souvent militaires (lance, heaume, gonfanon, etc) tandis que l'autre groupe contient la plupart des autres désignations. Les dames, quant à elles, forment un groupe assez à l'écart. Si l'on cherche une interprétation aux deux axes répartissant les modalités, on pourrait considérer l'axe 2 comme représentatif du niveau de richesse, avec en haut les désignations renvoyant à un certain niveau de pauvreté (joglars), puis un peu plus bas la simple cotte et les désignations de paubres cavalliers et de borges, et tout en bas, les vêtements et accessoires évoquant la richesse (le vair, la couronne, le diadème des trobairitz). L'axe 1, quant à lui, semble plutôt représenter un degré de noblesse, qui se caractériserait par des attributs militaires et chevaleresques (l'écu armorié et le carapaçon sont tout à la gauche du graphique, tandis que, de manière plus générale, les troubadours désignés comme nobles sont sur la gauche, et les autres sur la droite du graphique). Il ressort en effet une opposition entre les personnages participant de la noblesse et les autres, la noblesse formant un groupe relativement homogène et distinct.

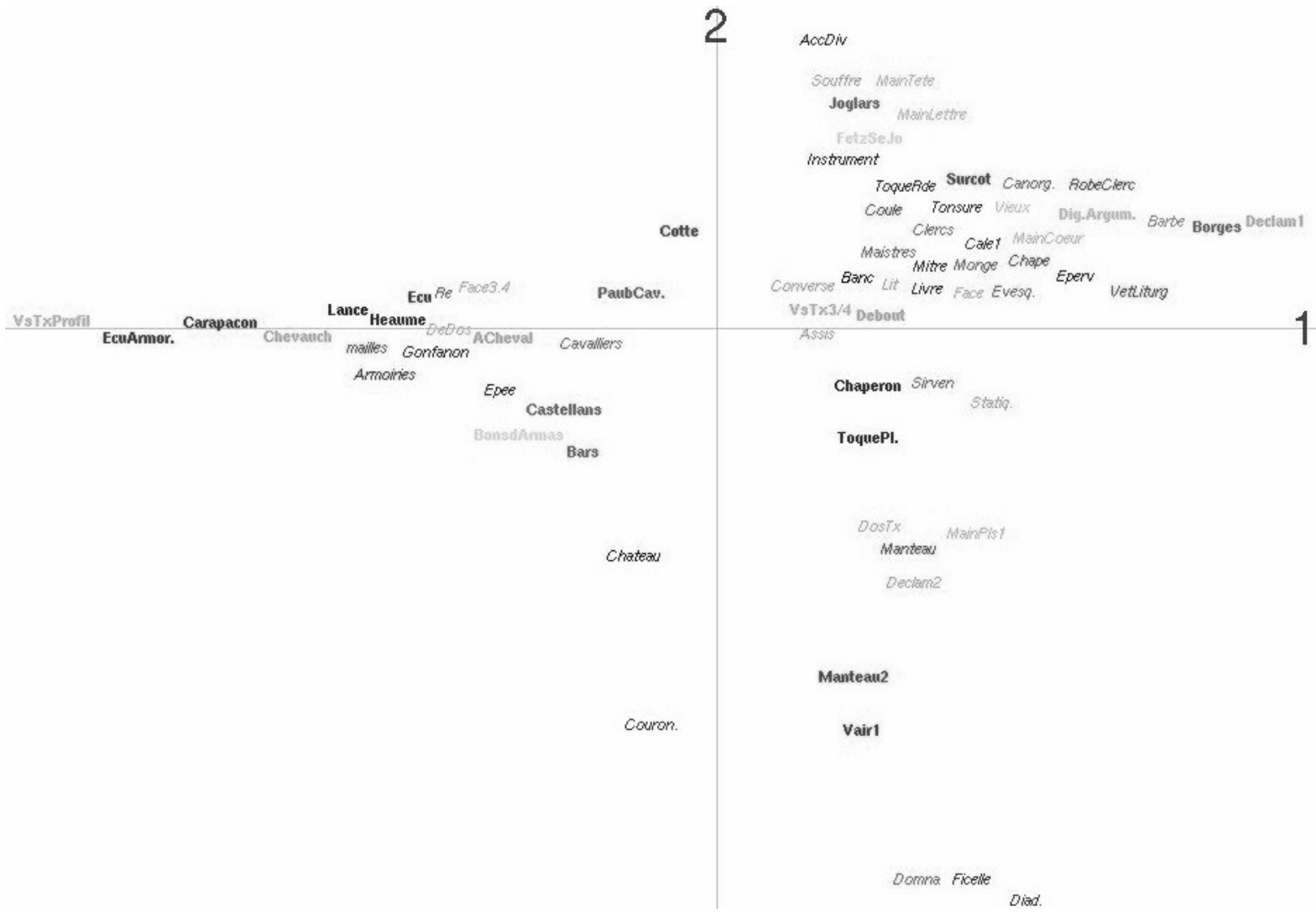

Fig. 1 : Miniatures de I, AFC, axes 1-2

L'examen de la répartition des points individus d'après leur désignation (fig. 2), nous mène à la même constatation de l'existence de deux groupes distincts. Ces graphiques ne permettent toutefois pas encore une analyse assez fine. 


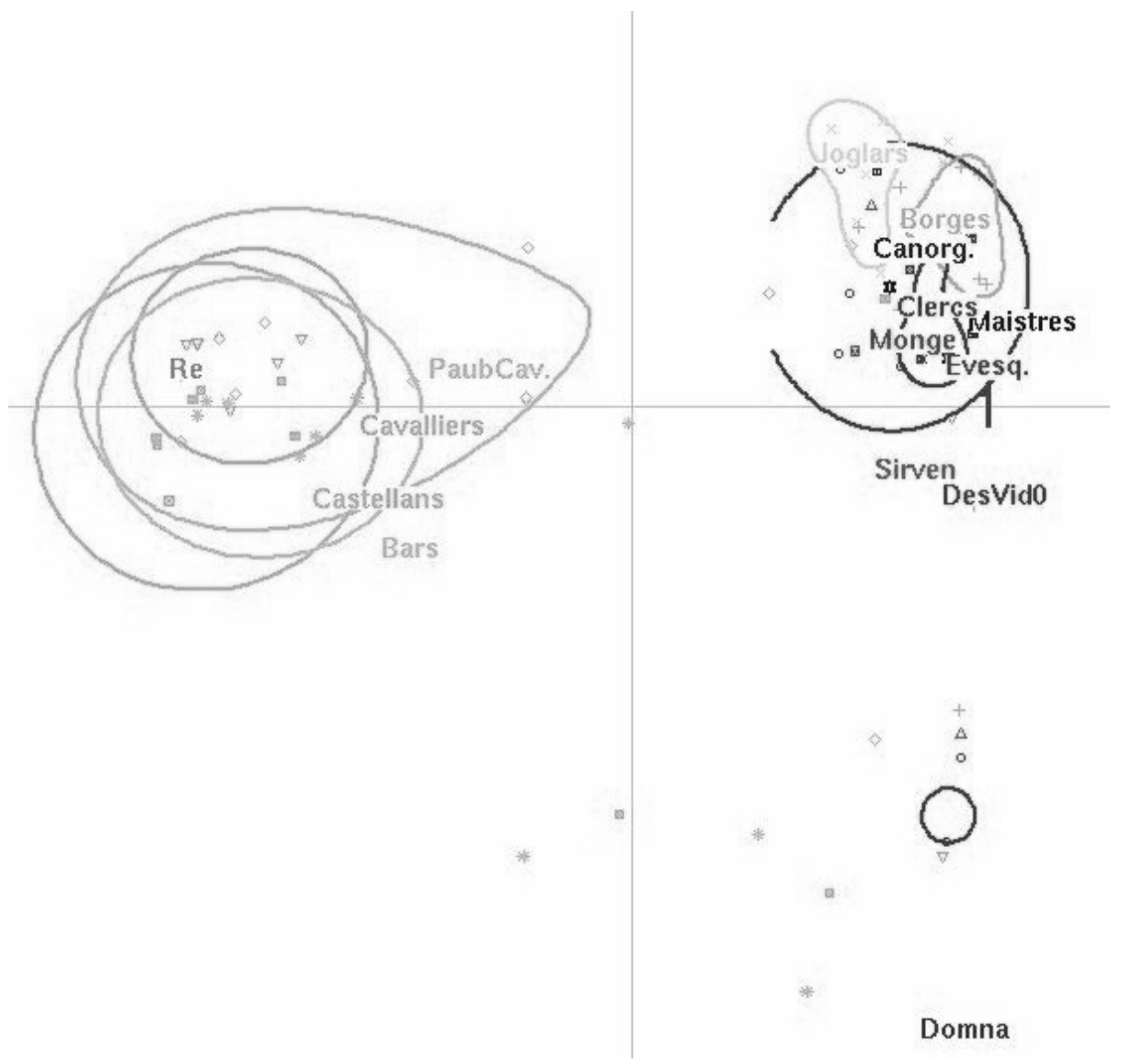

Fig. 2 : Points-individus d'après leurs désignations

Examinons maintenant individuellement les écarts à l'indépendance liant quelques caractères aux désignations des troubadours ${ }^{27}$. Si l'on examine tout d'abord les accessoires (fig. 3), qui paraissent l'attribut le plus évident, on peut observer encore une fois une séparation entre les nobles laïcs et les autres, fondée sur des accessoires guerriers (lances, épées...), mais on remarque également l'existence, chez les nobles, de deux groupes distincts. En effet, la lance à pennon armorié (gonfanon) semble être l'attribut des nobles les plus importants (castellans, bars) et des rois avec une certaine gradation.

27 Ces graphiques, représentant les écarts à l'indépendance, sont dits « de Bertin-Cibois » des noms de son inventeur, Jacques Bertin, et du sociologue Philippe Cibois qui en a fait grand usage. Les valeurs positives (attractions) s'élèvent au-dessus de la ligne et sont représentées en noir; les valeurs négatives (répulsions) descendent sous la ligne et sont en blanc. 


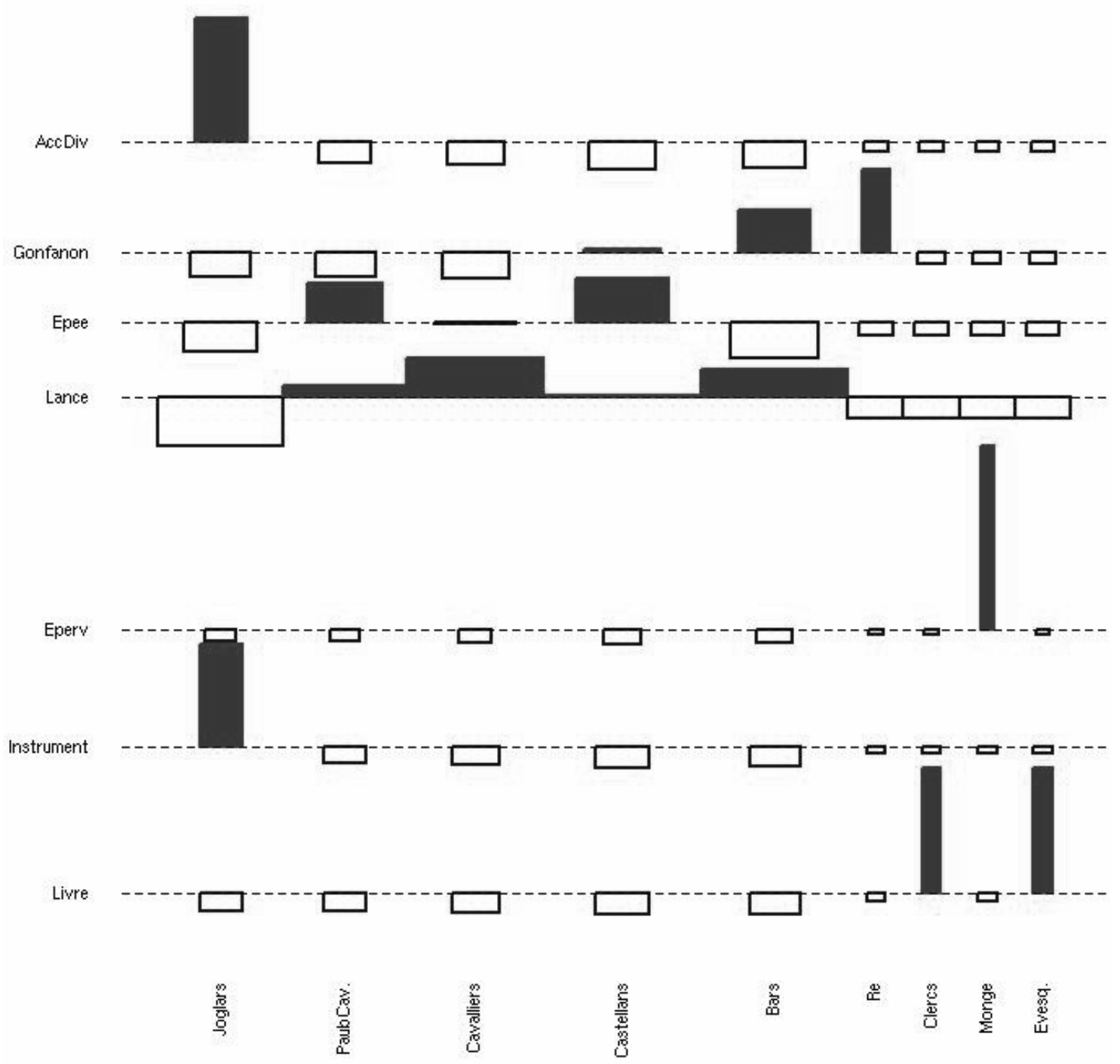

Fig. 3 : Les accessoires dans $I$

On retrouve, de façon très nette, cette répartition en deux sous-groupes, et cette gradation dans l'examen des armoiries, de l'écu et de la housse de cheval. Les armoiries semblent en effet être réservées au groupe des plus nobles. 


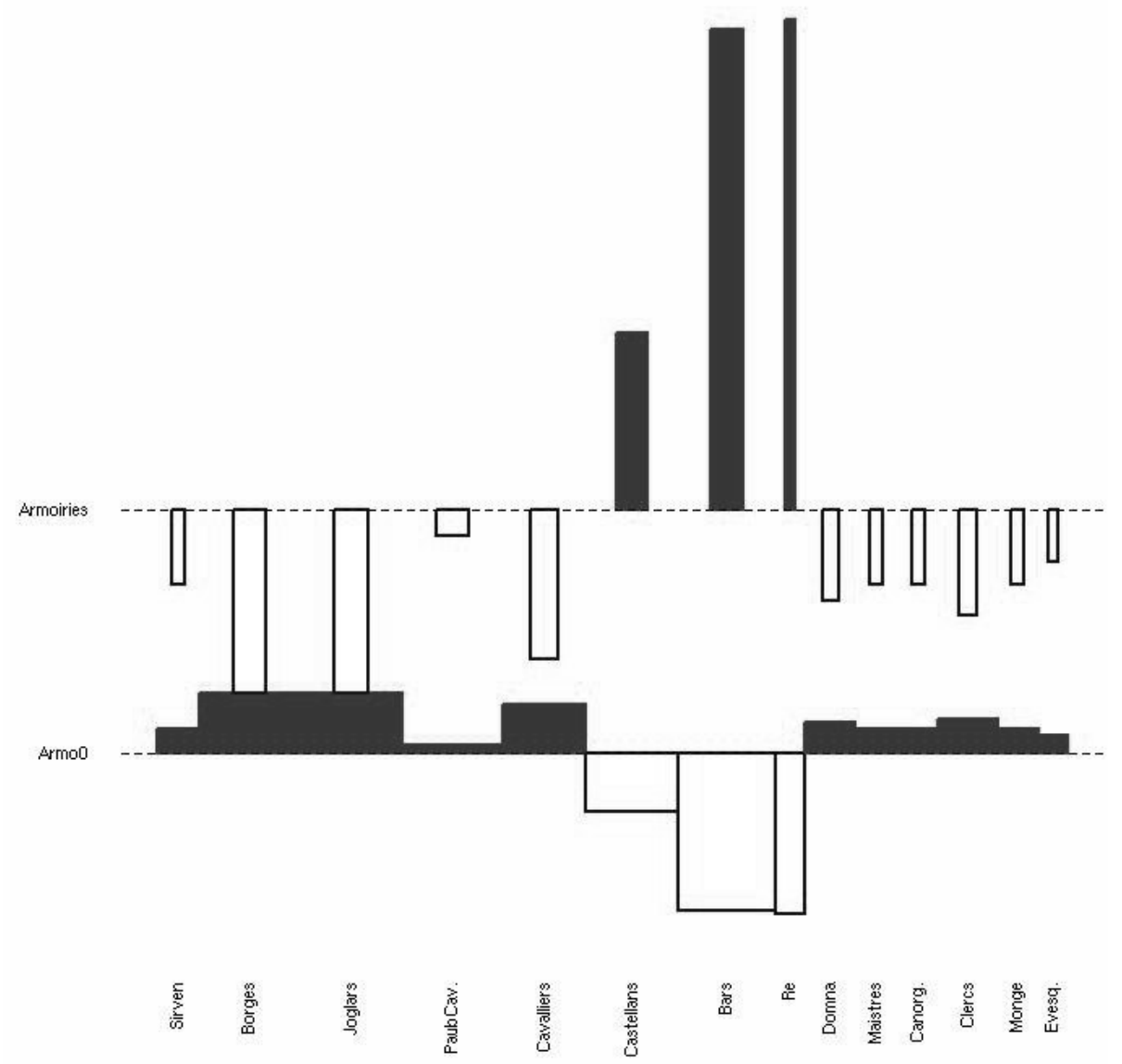

Fig. 4 : Les armoiries dans $I$

Du côté des formules employées, en prenant ici deux exemples caractéristiques, on retrouve cette division en deux groupes, ainsi que la gradation : la formule «bons cavalliers d'armas " s'applique à un groupe réunissant paubres cavalliers, cavalliers, castellans, barons et rois, avec une certaine gradation, tandis que la formule « fetz se joglars » se rencontre chez les autres, tant clercs que bourgeois notamment.

On peut donc dire que les personnages représentés en armes, clairement séparés des autres, se divisent en deux sous-groupes, réunissant d'un côté pauvres chevaliers et chevaliers, et de l'autre châtelains, barons et rois, et qu'au sein de cette répartition existe une gradation dans la noblesse, exprimée dans l'image par un vocabulaire comprenant armoiries, armes, ainsi que dans le texte par certaines formules comme « bons cavalliers d'armas ».

Il semblerait que, dans le chansonnier $I$, l'on soit face à une représentation de la noblesse qui se concentre sur des attributs chevaleresques et guerriers et qui prend le pas sur les représentations de noble à pied, déclamant. On ne rencontre qu'en quatre occasions des nobles à pied et sans armes, en train de déclamer, et ces exceptions peuvent être expliquées ${ }^{28}$. Il y a là peut-être une particularité du chansonnier $I$ par rapport aux chansonniers $A$ et $K$ dans lesquels cette tendance, quoique déjà présente, paraît moins affirmée et dans lesquels les représentations de nobles en train de déclamer sont peut-être légèrement plus fréquentes, mais qui surtout possèdent une gamme moins large d'attributs militaires. Le chansonnier $I$ est d'ailleurs le seul, parmi les trois pris en ligne de compte, à contenir la représentation d'une scène de combat, malgré les vœux de certaines postilles de $A^{29}$.

28 Ce sont Arnaut Daniel, Guiraut lo Ros, Raimbaut d'Aurenga, Sordel de Mantoue ; voir Lemaître et Vielliard (2006, p. 19, 103, 127, 173 et $n^{\circ} 9,54,68$ et 98).

29 Il s'agit des postilles concernant Guilhem de Cabestany et Aimeric de Peguilhan ; voir Lemaître et Vielliard 
Il devient plus important de matérialiser une différence de statut des personnages en fonction de leur rang, de transcrire une organisation hiérarchique de la noblesse, que de mettre en valeur le statut de troubadour, de poète, et c'est ainsi que pour le troubadour noble, son statut, son rang prend le pas sur l'activité poétique. Ce classement des troubadours, ici matérialisé par l'image, ne va pas pas toutefois, dans $I$ du moins, jusqu'à déterminer l'ordre des sections d'auteur, qui est plus un ordre de "dignité » des troubadours en tant qu'auteurs et ce contrairement à la structure d'un certain nombre de chansonniers de trouvères construits autour d'un ordre des auteurs qui place les trouvères les plus nobles en tête ${ }^{30}$, ou à certains chansonniers du Minnesang, comme le codex Manesse, qui s'ouvre sur le «Keiser Heinrich $»^{31}$ (Henri VI) à qui succèdent rois, marquis, ducs et comtes.

Jamais représentés en train de jouer d'un instrument, c'est là une activité de jongleur, les nobles du chansonnier $I$ ne le sont plus en train de déclamer, peut-être parce qu'au moment de la réalisation du chansonnier, composer et déclamer la lyrique occitane n'est plus l'affaire des seigneurs courtois, comme elle l'était encore quelque temps auparavant, mais celle de spécialistes et d'érudits. Il y a, en ce domaine, une certaine évolution qui est assez vraisemblablement celle de la perception des troubadours et de la lyrique occitane.

Pour terminer, je reprendrai une citation d'Angelica Rieger, qui faisait remarquer que « l'analyse de ces enluminures est (...) d'une grande complexité pour le philologue et elle lui demande à la fois un travail de paléographe, de codicologue, d'historien et d'historien de l'art ; et s'il doit parfois déplorer son dilettantisme dans ces domaines, il est en revanche mieux à même à tenir compte du rapport (...) entre le texte et l'image $»^{32}$. Pour ce sujet, comme pour d'autres, une approche interdisciplinaire est non seulement souhaitable, mais également incontournable.

École nationale des chartes (Paris)

Jean-Baptiste CAMPS

\section{Références bibliographiques}

Anglade, Joseph, 1924. « Miniatures des chansonniers provençaux », Romania 50, 593-604.

Boutière, Jean, Schutz, Alexander-Herman, 1964. Biographies des Troubadours. Textes provençaux des XIII et XIV ${ }^{e}$ siècles, Paris, A. G. Nizet.

Brunel-Lobrichon, Geneviève, 1991. «Iconographie du chansonnier provençal $R$ », Lyrique romane médiévale : la tradition des chansonniers (Actes du colloque de Liège 1989), Liège, Bibliothèque de la Faculté de Philosophie et Lettres de l'Université de Liège, 245272.

Camps, Jean-Baptiste, 2009. Vocabulaire du texte, vocabulaire de l'image : la représentation des troubadours dans les chansonniers occitans A (BAV, Vat. Lat. 5232), I (BnF, $F r$. 854) et $\mathrm{K}(B n F, F r$. 12473), Mémoire de Master II, dir. F. Vielliard et F. Zinelli, Paris, École nationale des chartes.

Folena, Gianfranco, 1990. " Tradizione e cultura trobadorica nelle corti e nelle città venete », Culture e lingue nel Veneto medievale, Padoue, 1-37 (initialement paru dans Storia della cultura veneta, I. dalle origini al trecento, Venise, 1976).

Huot, Sylvia, 1987. From song to book: the poetics of writing in old French lyric and lyrical narrative poetry, New-York, Cornell University Press, (compte rendu dans B.E.C., 149

(2008, p. 26 et 62). Il est vraisemblable que les souhaits de ces postilles aient parus inadaptés au miniaturiste de $A$ et s'éloignant trop des représentations traditionnelles.

30 Il s'agit des chansonniers $\mathrm{Pb4}, \mathrm{Pb6}, \mathrm{Pb8}, \mathrm{Pbl1}, \mathrm{Pb17}, \mathrm{Pa}, \mathrm{R}$; sur ce classement, voir Huot (1987).

31 Cod. Pal. germ. 848, f. 6 .

32 Rieger (1985, p. 286). 
(1991),169--172).

Jullian, Martine, 2007. « Images de trobairitz », Clio 25.

Kendrick, Laura, 2001. "L'image du troubadour comme auteur dans les chansonniers », Auctor et Auctoritas : invention et conformisme dans l'écriture médiévale, Paris, École des chartes, 508-519.

Lemaître, Jean-Loup, Vielliard, Françoise, et al., 2006. Portraits de troubadours : initiales des chansonniers provençaux I \& K, Ussel/Paris, De Boccard.

Lemaître, Jean-Loup, Vielliard, Françoise, et al., 2008. Portraits de troubadours : initiales du chansonnier provençal A, Cité du Vatican, Biblioteca Apostolica Vaticana, Studi e Testi 444.

Mariani-Canova, Giordana, 2004. «La miniatura », La pittura nel Veneto : le origini, Milan, Electa, 223-244.

Mariani-Canova, Giordana, 2008. « Il poeta e la sua immagine : il contributo della miniatura alla localizzazione e alla datazione dei canzonieri provenzali AIK e $N »$, I Trovatori nel Veneto e a Venezia Atti del Convegno Internazionale (Venezia, 28-31 ottobre 2004), Rome et Padoue, Antenore, 47-76.

Meneghetti, Maria-Luisa, 1984 et 1992. Il pubblico dei Trovatori : ricezione e riuso dei testi lirici cortesi fino al XIV secolo, Turin, G. Einaudi.

Rieger, Angelica, 1985. "Ins e l cor port, dona, vostra faisso. image et imaginaire de la femme à travers l'enluminure médiévale dans les chansonniers de troubadours », Cahiers de civilisation médiévale 28 , 385-415.

Wilson Poe, Elizabeth, 1984. From poetry to Prose in Old Provençal : The Emergence of the Vidas, the Razos and the Razos de Trobar, Birmingham, Summa publications.

Zinelli, Fabio, 2007. « Sur les traces de l'atelier des chansonniers occitans $I K$ : le manuscrit de Vérone, Biblioteca Capitolare, DVIII et la tradition méditerranéenne du Livres dou Tresor », Medioevo Romanzo 31, 7-69.

Zufferey, François, 1987. Recherches linguistiques sur les chansonniers provençaux, Genève, Droz. 